\title{
Goods distribution route optimization method for warehousing and logistics industry
}

\author{
Li jing
}

Qingdao Huanghai University, 266427

Keywords: logistics distribution; route selection; hybrid ant colony algorithm

\begin{abstract}
In the current study of goods distribution route optimization method in the industry of warehousing and logistics, because the current algorithm for route optimization have sluggish searching speed, and vulnerable to be involved in local optimal solution, a goods distribution route optimization method is raised for warehousing and logistics industry founded on hybrid ant colony algorithm. The method first carries on the detailed analysis for goods distribution route optimization, the mathematical model for optimizing goods distribution route is constructed, ant colony algorithm is brought up to resolve the model, and the quantum computation theory is incorporated to proceed quantum bit encoding for the pheromone on the route, quantum rotation gate and the optimal route are utilized to update the pheromone, and then effectively complete the optimization of goods distribution route for warehousing and logistics industry. Simulation results demonstrate that the goods distribution route optimization method based on hybrid ant colony algorithm for warehousing and logistics industry has strong global search ability and convergence speed, can excellently resolve the problem occurred in logistics distribution route.
\end{abstract}

\section{Introduction}

As the logistics industry is going through the rapid evolving stage, goods distribution route optimization has become a major problem to be solved immediately in the logistics industry [1.2.3]. The goods distribution route optimization method for warehousing and logistics industry can effectively solve the problem [4.5.6]. How to improve the reliability and credibility of logistics service ofthe enterprise appeals to a lot of experts and scholars [7.8]. As the goods distribution route optimization method for warehousing and logistics industry has comprehensive significance for expansion, it has become the focus topics being researched in the industry [9.10].

At present, the mainstream goods distribution route optimization method for warehousing and logistics industry based on the particle algorithm, the neural network algorithm, and also shuffled frog leaping algorithm. However, the current algorithm for route optimization have slow searching speed, and venerable to be involved in local optimal solution.

Considering the problems pronounced above, a goods distribution route optimization method is offered for warehousing and logistics industry founded on hybrid ant colony algorithm. The method has robust global searching capability and convergence speed, and can meritoriously resolve the problem occurred in logistics distribution route.

\section{Principle of goods distribution route optimization in warehousing and logistics industry}

During the optimization course of goods distribution route in warehousing and logistics industry, the load of freight vehicle and the volume of distribution vehicle are considered as main constraints for the model, the feasibility of resolving the model is emphasized, and the suitable optimization routeway is sorted out to shrink the time for optimization and the consumption of computing resources. The explicit steps are as follows:

During the optimization course of goods distribution route in warehousing and logistics industry, hypothesizing the mathematical model of optimized logistics distribution route is articulated as below:

$$
\min F=K \sum_{i=1}^{i} \sum_{r=1}^{r} x i r+\sum_{i=4}^{i} \sum_{r=1}^{r} x_{i r} l_{r}
$$


In the formula, $I$ is the number of warehouse, $R$ is the number of vehicles, $i$ is warehouse serial number, xir is the binary variables among $0 \sim 1$, if the vehicle $r$ is starting from the warehouse $i$, and ultimately coming back to the warehouse, $x i r=1$, else $x i r=0$.

In the optimization progress of goods distribution route, using the threshold value accepting criteria to receive poor solution, where $f\left(s^{\prime}\right)$ is the objective function of adjacent solution , $f(s)$ is the objective function value of the finest solution at this time, it can be drawn:

$$
\frac{f\left(s^{\prime}\right)-f(s)}{f(s)} \leq 1 \%
$$

\section{Implementation of goods distribution route optimization in warehousing and logistics industry}

3.1 The establishment of mathematical model of goods distribution route optimization

During the duration of optimizing goods distribution route for warehousing and logistics industry, assuming there are S customers, and the request and location of each customer are recognized, distribution enterprises arrange $\mathrm{K}$ cars from the distribution center to the customers' location in maximum, and , return logistics center after completing the task of distribution. The load of each vehicle is $10 \mathrm{t}$, the vehicle route have to be arranged so that the total cost (such as distance, time, etc.) is minimum, and satisfies the following conditions:

(1) the sum of customer demand on each line does not exceed the load of vehicles.

(2) the demand of each customer center must only be done by a car.

(3) the distribution line traverses all customers center.

During the duration of optimizing goods distribution route for warehousing and logistics industry, the entire amount of customer locations articulated as $s$, the demand of customer point $i$ is stated by the $q_{i}$, the distance from the customer point $\mathrm{i}$ to $\mathrm{j}$ customers is $d_{i j}$, K is the sum of vehicles, the maximum loading capacity of vehicle $\mathrm{K}$ expressed as $Q_{i}$, the total number of customer delivered by vehicle $\mathrm{L}$ is $n_{l}$. Then the constraints of goods distribution route are expressed as:

During the duration of optimizing goods distribution route for warehousing and logistics industry, the sum of customer demand on each line does not exceed the load of vehicles, i.e.: $\sum_{i=1}^{n k} q_{k}^{i}$

During the duration of optimizing goods distribution route for warehousing and logistics industry, the demand of each customer center must only be done by a car, i.e.: $A_{K 1} \cap A_{K 2}$

During the duration of optimizing goods distribution route for warehousing and logistics industry, the distribution line traverses all customers center, can be stated by the formula:

$$
\begin{gathered}
\cup_{k}^{k}=1 A_{K}=\{1,2, S\} \\
\sum_{i=1}^{n k} q_{k}^{i}
\end{gathered}
$$

During the duration of optimizing goods distribution route for warehousing and logistics industry, as the goods distribution cost and the transportation distance correlation is stronger, aiming to shorten the calculation, the transportation distance $\mathrm{Z}$ shortest as the optimization objective, the optimization model of logistics distribution route problem can be articulated as:

$$
\min z=\sum_{k=1}^{k}\left[\sum_{i=1}^{n k} d_{k}^{i}+d_{r}^{n}\right] \cdot \operatorname{sgn}(n k)
$$

3.2 The realization of goods distribution route optimization for warehousing and logistics industry

(1) In the optimization process of goods distribution route optimization for warehousing and logistics industry, the parameter $\alpha, \beta, \rho, \gamma$ are designated with certain value, the sum of ants is 
$\mathrm{m}$, the maximum quantity of the iterative times is $N_{M A X}$, the current iterations at $\mathrm{t}=0$, pheromone is $\tau_{i j}(0)=1$, information, in order to make all states occurred as the same probability when algorithm is searching initially, all $\alpha_{i j}$ and $\beta_{i j}$ values in the ants quantum pheromone code are $1 / \sqrt{2}$.

(2) In the optimization process of goods distribution route optimization for warehousing and logistics industry, $\mathrm{m}$ ants are placed in the logistics distribution center, each ant have a solution, referring the constraint conditions of logistics distribution, founded on the following equation to pick next customer, applied the state transition rule repeatedly, until the ant k completed distribution for all customers.

$$
j=\left\{\begin{array}{c}
\arg \max \left\{\tau_{i l}\left(\eta_{i l}\right)\right\} \\
j, \text { otherwise }
\end{array}\right.
$$

In the formula, $\tau_{i l}$ is the pheromone concentration along the route $(i, l), \eta_{i l}=1 / c_{i j}$ is the inspired value of distribution route $(i, l)$.

During the duration of optimizing goods distribution route for warehousing and logistics industry, the customer $j$ is a random variable generated based on the probability distribution given by the above formula with roulette method. With the formula to express:

$$
p_{i j}^{k}=\frac{\left[\tau_{i l}\right]^{\alpha}\left[\eta_{i l}\right]^{\beta}}{\sum_{i \in n_{i}^{k}}\left[\tau_{i l}\right]^{\alpha}\left[\eta_{i l}\right]^{\beta}}
$$

In the formula, $\alpha$ and $\beta$ are two parameters, which symbolize the relative influence of pheromone and heuristic information.

(3) In the optimization process of goods distribution route optimization for warehousing and logistics industry, if $\mathrm{m}$ ant constructed their own solution, then continue step 4, otherwise back to step 2.

(4) In the optimization process of goods distribution route optimization for warehousing and logistics industry, on the basis of the existing optimum solution, the quantum rotation gate criteria is employed to modernize quantum information probability amplitude of ants in each distribution route, the following equation is adopted to update pheromone as well.

$$
\begin{aligned}
& \tau_{i l}=\tau_{i l}+\sum_{k=1}^{m} \Delta \tau_{i l}{ }^{k} \\
& \tau_{i l}=(1-\rho) \tau_{i l}
\end{aligned}
$$

(5) In the optimization process of goods distribution route optimization for warehousing and logistics industry, if meet the end condition, i.e., $t \geq N_{\text {MAX }}$, yield the optimal solution, so as to get the preeminent scheme of logistics distribution route, or $t=t+1$, go to step 2 , continue to implement.

\section{Experiment and simulation}

In order to prove the authenticity and effectiveness of goods distribution route optimization method for warehousing and logistics industry based on the hybrid ant colony algorithm, there is the need to design an experiment. Assuming a company had a logistics distribution center, there were 5 goods transport vehicles (each vehicle load was 1 tons), the good has to be distributed to seven customer locations, individually, by using the improved algorithm and the traditional algorithm to solve the route optimization problem for continuous 70 times, diverse results were presented in table 1. 
Table 1 the comprehensive performance comparison of improved algorithm and the traditional algorithm

\begin{tabular}{lcc}
\hline Evaluation index & Improved algorithm & traditional algorithm \\
\hline $\begin{array}{c}\text { The average value of the } \\
\text { route } / \mathrm{KM}\end{array}$ & 209.55 & 220.77 \\
$\begin{array}{c}\text { The maximum value of the } \\
\text { route } / \mathrm{KM}\end{array}$ & 210.9 & 225.3 \\
$\begin{array}{c}\text { The minimum value of the } \\
\text { route } / \mathrm{KM}\end{array}$ & 198.6 & 228.6 \\
$\begin{array}{c}\text { the times of using } 70 \text { times } \\
\text { to find the optimal value } \\
\text { /times }\end{array}$ & 50 & 20 \\
\begin{tabular}{l} 
Average time consuming/s \\
\hline
\end{tabular} & 25 & 50 \\
\hline
\end{tabular}

It is obvious to see from Table 1, the improved algorithm have better performance on searching minimum value and higher efficiency compared to the traditional algorithm, and the reliability of optimization is robust, so as to complete the optimization of goods distribution route for warehousing and logistics industry effectively.

\section{Conclusions}

As the current algorithm for route optimization have sluggish searching speed, and vulnerable to be involved in local optimal solution, a goods distribution route optimization method is raised for warehousing and logistics industry founded on hybrid ant colony algorithm. The method first carries on the detailed analysis for goods distribution route optimization, the mathematical model for optimizing goods distribution route is constructed, ant colony algorithm is brought up to resolve the model, and the quantum computation theory is incorporated to proceed quantum bit encoding for the pheromone on the route, quantum rotation gate and the optimal route are utilized to update the pheromone, and then effectively complete the optimization of goods distribution route for warehousing and logistics industry. Simulation results demonstrate that the goods distribution route optimization method based on hybrid ant colony algorithm for warehousing and logistics industry has strong global search ability and convergence speed, can excellently resolve the problem occurred in logistics distribution route.

\section{References}

[1] Wang Mengnan, She Qian Fang. Warehouse goods ABC classification based on EIQ method [J]. Value engineering, 2013, (25): 17-19.

[2] Wang Xiaoyan, Ma Xin. Thinking about distribution system construction of Gansu electric power storage materials [J]. Gansu metallurgy, 2013, 35 (1): 124-126.

[3] Yu Yong, Wei Feng. The application of database technology in the warehouse storage of goods [J]. Logistics technology, 2014, (10): 463-464.

[4] Sun Yongling. The development of school-based curriculum practice of warehousing distribution practices based on work process, in Shandong Province, Wendeng normal school as an example of management [J]. New campus, 2014, (9): 76-76.

[5] Lu Qiankun. Warehouse distribution optimization of the national grid net in northern Hebei Electric Power Co. [J]. Modern commerce industry, 2014, 26 (7): 168-168.

[6] Xiang Xin. The suggestion of development of green warehousing [J]. Logistics technology and application, 2014, 19 (6): 56-57.

[7] Xiong Naiping. Curriculum reform of <warehousing and distribution $>$ of logistics Service and 
management major in vocational education school [J]. In mid - new course of study, 2013, (12): 126-127.

[8] Gao Zhan. Research on decision making model of hybrid warehousing mode in tobacco distribution center [J]. Logistics technology, 2013, 32 (6): 88-90.

[9] Bai Yanhui, Zhao Ruxiong, Ran Maoxuan. Application of dense storage technology in Qinhuangdao cigarette logistics distribution center [J]. Logistics technology and application, 2013, (5): 66-69.

[10] Zhang Peng, Wang Luda, Hu Dan. Research of multi objective optimization based on quantum genetic algorithm [J]. Computer simulation, the ant colony 2013, 30 (4): 322-325. 